\title{
Recurrent gastrointestinal bleeding associated with chronic pancreatitis
}

\author{
A P Jenkins, M M El-Omar, J C L Booth, A K Banerjee, K G Burnand, R P H Thompson
}

\begin{abstract}
A 52 year old man with chronic pancreatitis presented with recurrent upper gastrointestinal bleeding. Gastroscopy was normal, but visceral angiography suggested that there were gastric varices. Despite treatment with propranolol he had further episodes of bleeding and so underwent splenectomy to decompress the gastric varices. When the spleen was removed, however, an inflammatory mass in the head of the pancreas adherent to the posterior gastric wall was noted. Within it the splenic artery was visible and communicated with the gastric lumen through a small opening in the gastric wall. The artery was ligated and the patient has since had no further bleeding. Thus, chronic pancreatitis should be considered as a cause of recurrent upper gastrointestinal bleeding, especially when gastroscopy is normal.

(Gut 1995; 36: 314-316)
\end{abstract}

Keywords: gastrointestinal haemorrhage, pancreatitis.

The association between chronic liver disease and upper gastrointestinal bleeding is well known, and endoscopic evidence of varices, congestive gastropathy or ulcers can make the diagnosis easy. Chronic pancreatitis, on the other hand, is less well recognised as a cause of recurrent gastrointestinal bleeding and there are seldom abnormal findings on routine endoscopy. We describe a patient who had recurrent episodes of upper gastrointestinal bleeding and repeatedly normal gastroscopies, in whom chronic pancreatitis was eventually diagnosed as the cause of the bleeding.

\section{Case report}

The 52 year old master of a Cross Channel ferry was admitted to his local hospital in September 1991 after a large, fresh haematemesis. He had no previous history of gastrointestinal bleeding but in 1987 chronic pancreatitis had been diagnosed from an endoscopic retrograde pancreatogram; the aetiology was probably alcohol abuse, as he was then drinking six units of alcohol/day. Gastroscopy did not identify a source for the bleeding and he was given omeprazole $20 \mathrm{mg}$ every day before discharge. In October 1991, however, he was readmitted after an episode of melaena, for which he required transfusion of six units of blood. Barium enema and sigmoidoscopy were normal, but he continued to experience minor episodes of melaena and again required blood transfusion. He was therefore referred for further investigation to the Dreadnought Seamen's Unit at St Thomas' Hospital.

On admission he was well. Investigations showed: haemoglobin $84 \mathrm{~g} / \mathrm{l}$, white blood cell count $5 \cdot 3 \times 10^{9} / 1$ and platelet count $402 \times 10^{9} / 1$. Coagulation studies and liver blood tests were normal as were gastroscopy, barium enema, total colonoscopy, and abdominal ultrasonography. Coeliac axis angiography showed a normal arterial phase, but in the venous phase there was no filling of the splenic vein with instead enlarged varicose collateral veins draining the spleen into the superior mesenteric vein (Figure). A diagnosis of gastric varices was made and he was given propranolol $40 \mathrm{mg}$ thrice daily and discharged. He experienced no further gastrointestinal bleeding for nine months and the haemoglobin concentration remained normal. Because of considerable fatigue, however, the dose of propranolol was reduced to $40 \mathrm{mg}$ twice daily.

In August 1992 he was readmitted locally after a further haematemesis. Gastroscopy was again normal and he was transfused four units of blood. The dose of propranolol was increased again to $40 \mathrm{mg}$ thrice daily, but he had another episode of melaena necessitating a further admission. He was therefore transfused an additional four units and transferred back to St Thomas' Hospital in September 1992 where a further gastroscopy showed fresh blood in the stomach but no endoscopic evidence of gastric varices. He continued to pass fresh melaena necessitating another transfusion and so underwent laparotomy with a view to splenectomy on 22 September.

At laparotomy the spleen was not enlarged and no gross collaterals were noted around the stomach, but after splenectomy had been performed it was apparent that there was a mass in the head of the pancreas adherent to the posterior aspect of the stomach high on the lesser curve. The stomach was mobilised off the pancreas and the splenic artery was then visible in the base of the pancreatic mass, with a small opening in the gastric wall communicating with the artery. The splenic artery was underrun, the opening in the gastric wall closed, and a truncal vagotomy and pyloroplasty performed.

$\mathrm{He}$ has had no further episodes of gastrointestinal bleeding for 12 months. A subsequent computed tomogram has shown a $2 \mathrm{~cm}$ diameter pseudocyst in the head of the pancreas.

\section{Discussion}

The cause of upper gastrointestinal haemorrhage remains undiagnosed in between 5 and
London SE1 7 Hospita

Accepted for publication 6 May 1994 

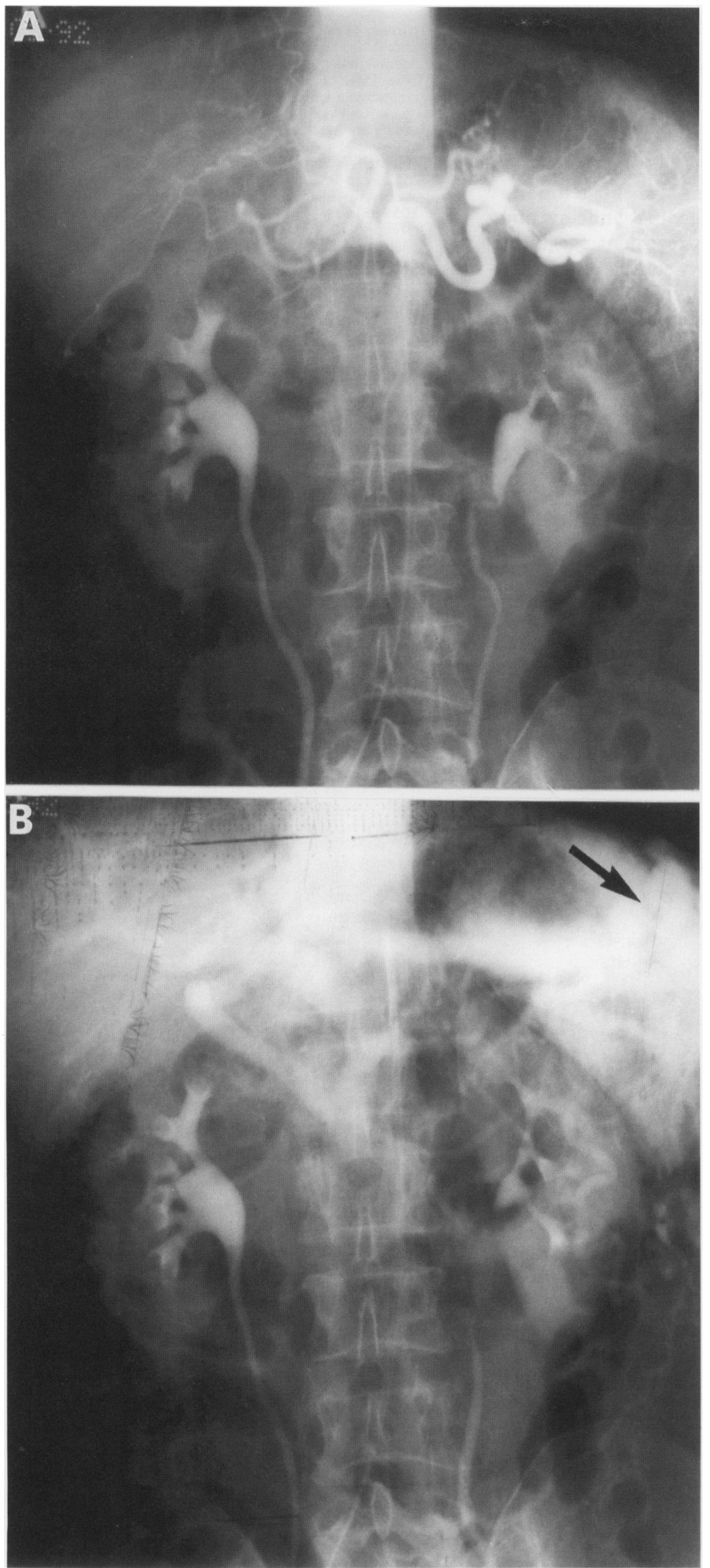

(A) Arterial phase of coeliac angiogram showing normal splenic and hepatic arteries; (B) venous phase of coeliac angiogram showing enlarged varicose collateral veins (indicated by an arrow) draining the spleen by the gastric veins into the superior mesenteric and portal veins. No filling of the splenic vein is seen.

$10 \%$ of patients after initial gastroscopy. ${ }^{1}$ Especially in the presence of blood, lesions of the oesophagus, stomach, and duodenum may be missed; conversely, lesions such as Dieulafoy's gastric vascular abnormality or bleeding from the bile or pancreatic ducts may only be diagnosed if they are actively bleeding at the time of endoscopy. A few sources of upper gastrointestinal bleeding are inaccessible to routine endoscopic inspection, such as vascular abnormalities of the small intestine, bleeding from a Meckel's diverticulum or an aortoenteric fistula.

Both acute and chronic pancreatitis are recognised causes of endoscopy negative upper gastrointestinal bleeding. The most common reason is probably bleeding into a pseudocyst. As a pseudocyst enlarges small vessels in its wall may rupture with bleeding into the cyst and, thence, if there is communication between the cyst and pancreatic duct, into the duodenum through the pancreatic duct. This is usually venous bleeding and not severe. ${ }^{2}$ Alternatively, a pseudocyst may erode a peripancreatic artery, most commonly the splenic artery. ${ }^{3}$ The resulting haemorrhage into the pseudocyst may be contained initially as a pseudoaneurysm before there is bleeding into the pancreatic duct and duodenum. Occasionally an enlarging pseudoaneurysm may rupture into adjacent gut or even the peritoneal cavity. ${ }^{3}$ Pseudoaneurysms can also occasionally form in the absence of pseudocysts by direct erosion of adjacent arteries by the inflammatory process within the pancreas. ${ }^{34}$

The other important cause of bleeding in chronic pancreatitis is thrombosis of the splenic and less commonly portal vein, which lie posteriorly to the pancreas. ${ }^{56}$ Splenic vein thrombosis causes 'left sided' or regional portal hypertension, which typically presents with splenomegaly and gastric varices, formed by the short gastric veins, often in the absence of oesophageal varices. The gastric varices are usually in the gastric fundus and around the cardia and their diagnosis can be difficult because they resemble rugal folds at endoscopy, which was thought to be the explanation for why they were not visualised in our patient.

Visceral angiography is the method of choice for investigating gastrointestinal bleeding from a suspected pseudoaneurysm associated with pancreatitis. ${ }^{78}$ If angiography is continued into the venous phase thrombosis of the splenic or portal vein can be diagnosed and gastric varices shown. This was so in this case, although the bleeding from the splenic artery into the stomach was not shown, presumably because there was no active bleeding at the time of the investigation. In retrospect it seems probable that the radiological diagnosis of gastric varices in our patient may have been an incidental finding. The varices were probably small, because they were not seen at endoscopy, nor was there evidence from ultrasonography or laparotomy of severe 'left sided' portal hypertension. It is, therefore, more likely that the major source of bleeding was the splenic artery through its communication with the stomach. Thus, although visceral angiography can be helpful in diagnosing the cause of bleeding associated with chronic 
pancreatitis, in our case the findings were misleading and delayed definitive diagnosis.

Pseudoaneurysms may be treated by surgical ligation of the vessels accompanied, if necessary, by drainage of a pseudocyst or local pancreatic resection. ${ }^{39}$ Angiographic embolisation has been used for pancreatic pseudoaneurysms either as a temporary manoeuvre to control bleeding while awaiting surgery or as definitive treatment for bleeding. 1011 Patients in whom angiographic treatment has been used will often require subsequent surgical treatment for a pseudocyst. For gastric varices, local endoscopic injection of the varices with tissue adhesive or thrombin may be effective. ${ }^{12}$ Treatment with propranolol may prevent rebleeding, but splenectomy may be needed to decompress the short gastric veins.

In conclusion, patients with chronic pancreatitis occasionally present with recurrent gastrointestinal bleeding. As our case showed, arterial haemorrhage in these patients can result from the involvement of adjacent arteries by the inflammatory process in the pancreas, but variceal bleeding as a result of 'left sided' portal hypertension must also be considered as a cause of severe haemorrhage.
1 Stevenson GW, Cox RR, Roberts CJC. Prospective comparison of double-contrast barium meal examination and fibreoptic endoscopy in acute upper gastrointestina haemorrhage. $B M \mathcal{F}$ 1976; 2: 723-4.

2 Morse JMD, Reddy KR, Thomas E. Hemosuccus pancreaticus: a cause for obscure gastrointestinal bleeding diagnosis by endoscopy and successul management by diagnosis by endoscopy and successful management by total parer

3 El Hamel A, Parc R, Adda G, Bouteloup PY, Huguet C, Malafosse M. Bleeding pseudocysts and pseudoaneurysms in chronic pancreatitis. Br $\mathcal{f}$ Surg 1991; 78: 1059-63

4 De Filippi UJ, Vargish T, Block GE. Massive gastrointestinal hemorrhage in pancreatitis secondary to visceral artery aneurysms. Am Surg 1992; 53: 616-21.

5 Little AG, Moosa AR. Gastrointestinal haemorrhage from left-sided portal hypertension: an unappreciated complication of pancreatitis. Am $\mathcal{F}$ Surg 1981; 141: 153-8.

6 Warshaw AL, Jin G, Ottinger LW. Recognition and clinical implications of mesenteric and portal vein obstruction in chronic pancreatitis. Arch Surg 1987; 122: 410-5.

7 Reuter SR, Redman HC, Joseph RR. Angiographic findings in pancreatitis. $A f R$ 1969; 107: 56-64.

8 Walter JF, Chuang VP. Bookstein JJ, Reuter SR, Cho KJ, Pulmaro CM. Angiography of massive hemorrhage secondary to pancreatic disease. Diagn Radiol 1977; 124: 337-42.

9 Bresler L, Boissel P, Grosdidier J. Major hemorrhage from pseudocysts and pseudoaneurysms caused by chronic pancreatitis: surgical therapy. World f Surg 1991; 15: 649-53.

10 Huizing WKJ, Kalideen JM, Bryer JV, Bell PSH, Baker LW. Control of major haemorrhage associated with pancreatic pseudocysts by transcatheter arterial embolization. pseudocysts by transcath

11 Steckman ML, Dooley MC, Jacques PF, et al. Major gastrointestinal hemorrhage from peripancreatic blood vessels in pancreatitis: treatment by embolotherapy. Dig Dis Sci 1984; 29: 486-97.

12 Westaby $\mathrm{D}$. The management of active variceal bleeding. f Hepatol 1993; 17: S34-7. 